\title{
Quantitative Resting State Electroencephalography in Patients with Schizophrenia Spectrum Disorders Treated with Strict Monotherapy Using Atypical Antipsychotics
}

\author{
Takashi Ozaki ${ }^{1,2}$, Atsuhito Toyomaki ${ }^{1}$, Naoki Hashimoto', Ichiro Kusumi ${ }^{1}$ \\ ${ }^{1}$ Department of Psychiatry, Graduate School of Medicine, Hokkaido University, ${ }^{2}$ Department of Psychiatry, Hokkaido Prefectural Koyogaoka \\ Hospital, Sapporo, Japan
}

\begin{abstract}
Objective: The effect of antipsychotic drugs on quantitative electroencephalography (EEG) has been mainly examined by the administration of a single test dose or among patients using combinations of other psychotropic drugs. We therefore investigated the effects of strict monotherapy with antipsychotic drugs on quantitative EEG among schizophrenia patients. Methods: Data from 2,364 medical reports with EEG results from psychiatric patients admitted to the Hokkaido University Hospital were used. We extracted EEG records of patients who were diagnosed with schizophrenia spectrum disorders and who were either undergoing strict antipsychotic monotherapy or were completely free of psychotropic drugs. The spectral power was compared between drug-free patients and patients using antipsychotic drugs. We also performed multiple regression analysis to evaluate the relationship between spectral power and the chlorpromazine equivalent daily dose of antipsychotics in all the patients.

Results: We included 31 monotherapy and 20 drug-free patients. Compared with drug-free patients, patients receiving antipsychotic drugs demonstrated significant increases in theta, alpha and beta power. When patients taking different types of antipsychotics were compared with drug-free patients, we found no significant change in any spectrum power for the aripiprazole or blonanserin groups. Patients taking risperidone demonstrated significant increases in alpha and beta power. Patients taking clozapine and olanzapine demonstrated significant slow wave increases. Multiple regression analysis revealed that the chlorpromazine equivalent dose was positively associated with theta power.

Conclusion: Use of any antipsychotic drug by patients was associated with a dose-dependent increase in theta power. However, each type of antipsychotic demonstrated different spectral power changes.
\end{abstract}

KEY WORDS: Antipsychotic aents; Electroencephalography; Gamma rhythm; Theta rhythm; Schizophrenia.

\section{INTRODUCTION}

Quantitative electroencephalography (EEG), which is a spectral power analysis method of the delta, theta, alpha, beta, and gamma wave bands, is a non-invasive neuroelectric method that has been widely used in patients to examine the pathophysiology of schizophrenia because psychoses have a neurobiological basis [1]. Although quantitative EEG results of schizophrenia patients can be affected by various factors such as age, sex, medication,

Received: June 30, 2020 / Revised: August 12, 2020

Accepted: August 13, 2020

Address for correspondence: Naoki Hashimoto

Department of Psychiatry, Graduate School of Medicine,

Hokkaido University, North 15 West 7, Sapporo 060-8648, Japan

E-mail: hashinao@med.hokudai.ac.jp

ORCID: https://orcid.org/0000-0001-8311-7148 and disease onset [2], the usefulness of quantitative EEG as a tool to assist in the diagnosis of patients and prediction of therapeutic effectiveness of treatments has been repeatedly reported in previous papers [3-7].

Antipsychotic drugs have produced relatively consistent effects on quantitative EEG findings in previous reports [8], but these findings are mainly based on administering antipsychotics to healthy subjects [5,9-11] or patients with chronic schizophrenia [7]. In normal healthy subjects, the use of sedative, low-potency neuroleptics (such as chlorpromazine) is characterized by increases in delta and theta power and decreases in alpha and beta power [7]. However, non-sedative, high-potency neuroleptics (such as haloperidol) are characterized by increases in alpha and beta power $[7,12]$. For haloperidol, the changes

() This is an Open-Access article distributed under the terms of the Creative Commons Attribution Non-Commercial License (http://creativecommons.org/licenses/by-nc/4.0) which permits unrestricted non-commercial use, distribution, and reproduction in any medium, provided the original work is properly cited. 
in the power spectrum vary according to medication status, dose, and responder/non-responder group [13-15]. In addition, some reports examining the chemical structure have indicated that psychotropic tricyclic compounds may be associated with the delta and theta power [12].

The effects of atypical antipsychotic drugs on quantitative EEG findings among schizophrenia patients have mainly been reported for those taking clozapine $[1,7,16,17]$, resulting in widespread increases in delta and theta power [1] and alteration of fast alpha power, especially in the treatment-responsive group [7]. Similar results were reported by a Korean group using multiple linear regression analysis, which showed increases in delta and theta power and a decrease in beta power [18]. One report discussed the effects of clozapine, olanzapine, and risperidone using low-resolution brain electromagnetic tomography analysis under strict antipsychotic monotherapy conditions [17]. The authors further reported that patients with schizophrenia receiving any of the drugs listed above demonstrated increases in delta and theta power in the fronto/temporo/occipital region and in alpha and beta power in the temporo/occipital region. Patients receiving clozapine demonstrated increases in delta and theta power in the frontal region, while patients receiving risperidone demonstrated no change in the power spectrum, suggesting that the effects on quantitative EEG differ for each type of antipsychotic drug.

The effect of antipsychotic drugs on the gamma band has been recently described in a limited number of reports. The gamma band is considered to be deeply associated with mental disorders and cognitive functions including several sensory modalities [19]. One report describing an atypical antipsychotic effect in low gamma-evoked oscillations in patients suggested that atypical antipsychotics normalize $30-50 \mathrm{~Hz}$ low gamma power [20]. However, another study examining the effect of atypical antipsychotic drugs on the resting state in schizophrenia patients found no significant change compared with healthy controls [4].

These previous reports investigating the effect of psychotropic drugs on quantitative EEG findings mainly examined the administration of a single test dose in patients with schizophrenia and healthy controls [7] or included patients using combinations of other psychotropic drugs such as other antipsychotics, benzodiazepines, and mood stabilizers, which may cause the results to be ambiguous
[18]. Additionally, the effect of antipsychotics on gamma power has not been well investigated. Furthermore, the effects of new antipsychotic drugs, such as blonanserin, which is mainly used in eastern Asian countries, have not been examined [21].

In this study, we hypothesized that the effect of atypical antipsychotics differs in each power band and that different types of antipsychotic drugs may show varying effects in patients with schizophrenia. We also hypothesized that these effects might be dose-dependent. Hence, in this study, we investigated the effects of strict monotherapy with antipsychotic drugs on quantitative EEG results among schizophrenia patients.

\section{METHODS}

\section{Participants}

A data bank of 2,364 EEG medical reports recorded from psychiatric patients admitted to the Hokkaido University Hospital in Hokkaido, Japan between January 2010 and October 2019 was used.

From this data set, we extracted EEG data of patients who were diagnosed with schizophrenia, schizotypal, delusional, and other non-mood psychotic disorders (F20-25 according to the International Classification of Diseases, tenth edition [ICD-10] [22]) and who were either undergoing strict antipsychotic monotherapy (without use of any associated psychotropic drug, including anticonvulsants, lithium, benzodiazepines, antidepressants, or anticholinergics) or were completely free of psychotropic drugs at the date of EEG recording. All patients were diagnosed by senior psychiatrists with at least 5 years of experience according to ICD-10 code. Every patient included in this study underwent either strict monotherapy or drug free for at least 3 days or more before the EEG recording. The exclusion criteria were age less than 13 years old, epilepsy, brain tumor, stroke, traumatic brain injury, previous drug overdose leading to coma, and treatment with electroconvulsive therapy in the 4 months before the EEG recording. We further excluded two patients who were using typical antipsychotic agents (sulpiride and levomepromazine).

The data collected from the medical records included sex, age, medication, disease onset, Global Assessment of Functioning (GAF) score, chlorpromazine equivalent daily dose of an antipsychotic drug (CP eq., mg/day), and Positive and Negative Syndrome Scale score [23]. 


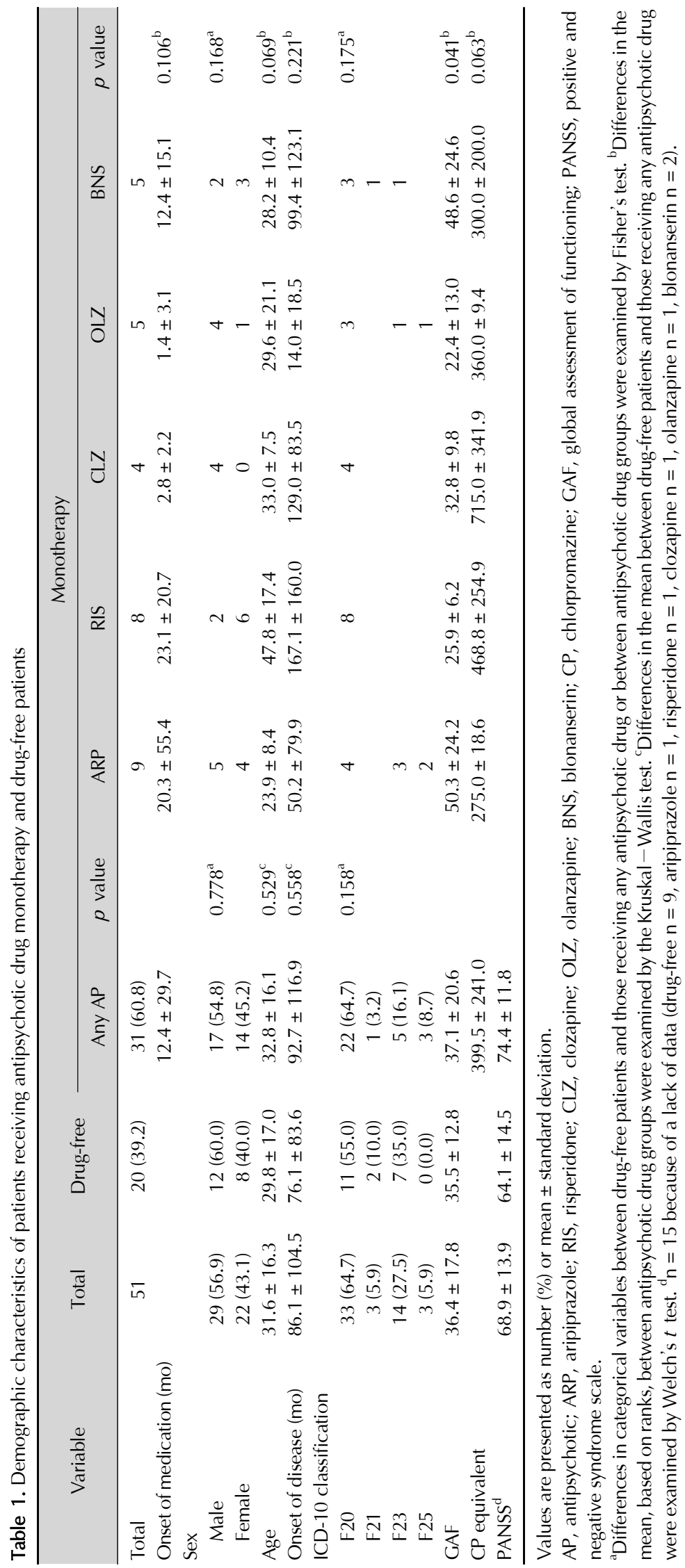


This study was approved by the Ethical Committee of the Hokkaido University Graduate School of Medicine (no. 020-0062).

\section{EEG Recordings}

The EEG recordings were performed according to the recommendations of the American Society for Electroencephalography, with the standard 10-20 system of electrode placement [24], using a digital EEG instrument (Neurofax EEG-1200; Nihon-Kohden, Tokyo, Japan).

The ipsilateral earlobes were used as a reference for all EEG electrodes. $\mathrm{Ag} / \mathrm{AgCl}$ electrodes were used, and the impedance was maintained below $5 \mathrm{k} \Omega$. The EEG data were filtered (time constant, 0.16 seconds; high-frequency filter, $100 \mathrm{~Hz}$ ) and digitized (sampling rate, $500 \mathrm{~Hz}$ ). The recordings were performed in a sound-attenuated room that had subdued lightning, and patients were in a semirecumbent position with their eyes closed but in a maximally alert state. The alertness of patients was controlled during the recording. If patterns of drowsiness appeared in the EEG recordings, the subjects were aroused by acoustic stimulation.

\section{Spectral Analysis}

We extracted a minimum of 60 seconds of quantitative EEG data that did not contain any artifacts. We performed this processes visually using Brain Electrical Source Analysis (BESA) 5.2 software (MEGIS Software, Grafelfing, Germany). We imported this one epoch EEG data into EEGLAB tool- box and then performed frequency analysis. We used the "spectopo" function of the EEGLAB toolbox. Spectral power was computed with a window of 60 s using Fourier transform. Each spectral power was estimated in frequency bands as follows: delta $(1-3 \mathrm{~Hz})$, theta $(4-7 \mathrm{~Hz})$, alpha $(8-13 \mathrm{~Hz})$, beta $(14-29 \mathrm{~Hz})$, and gamma (30100 Hz). MATLAB-2014 (The MathWorks, Natick, MA, USA) and EEGLAB were used for time frequency analysis.

\section{Statistical Analysis}

Demographic differences between patients in each antipsychotic drug group were examined using Fisher's exact test for categorical variables or the Kruskal-Wallis test for continuous variables. Comparisons of the spectral power between patients in each antipsychotic drug group and drug-free patients were performed using a MannWhitney $U$ test with Dunn's correction to adjust the $p$ values for multiple comparisons. We calculated Pearson's correlation coefficients between all band powers and the $\mathrm{CP}$ eq. of all patients, including drug-free patients (CP eq. $0 \mathrm{mg}$ ). The correlation between spectral power and CP eq. was analyzed using a multiple linear regression model to adjust for age, sex, and disease onset. Statistical analysis was performed using $\mathrm{R}$ version 3.2.3 [25].

\section{RESULTS}

\section{Demographic Characteristics}

As shown in Table 1, patients in each antipsychotic

Table 2. Comparison of each spectral power between patients receiving any antipsychotic drug and drug-free patients

\begin{tabular}{|c|c|c|c|c|c|c|c|c|}
\hline Lead & F3 & $\mathrm{F} 4$ & $\mathrm{C} 3$ & $\mathrm{C} 4$ & P3 & P4 & $\mathrm{O} 1$ & $\mathrm{O} 2$ \\
\hline \multicolumn{9}{|l|}{ AP } \\
\hline Delta & $54.3 \pm 4.0$ & $54.1 \pm 3.9$ & $52.9 \pm 3.3$ & $53.1 \pm 3.3$ & $53.1 \pm 3.2$ & $53.3 \pm 3.3$ & $53.2 \pm 4.1$ & $53.1 \pm 3.9$ \\
\hline Theta & $50.9 \pm 2.9^{*}$ & $50.8 \pm 2.9^{*}$ & $50.3 \pm 2.8^{* *}$ & $50.3 \pm 2.8^{* *}$ & $50.0 \pm 2.7^{* *}$ & $50.2 \pm 2.9^{*}$ & $49.9 \pm 3.5^{*}$ & $49.8 \pm 3.6^{*}$ \\
\hline Alpha & $49.4 \pm 2.8^{*}$ & $49.4 \pm 2.8^{*}$ & $49.5 \pm 3.0^{*}$ & $49.7 \pm 3.0^{*}$ & $50.6 \pm 3.5^{*}$ & $50.5 \pm 3.5^{*}$ & $51.7 \pm 4.6^{*}$ & $51.5 \pm 4.9$ \\
\hline Beta & $42.4 \pm 3.0$ & $42.4 \pm 3.0$ & $42.3 \pm 3.0^{*}$ & $42.6 \pm 3.0^{*}$ & $42.6 \pm 3.1^{*}$ & $42.6 \pm 3.0^{*}$ & $42.9 \pm 3.5$ & $43.0 \pm 3.6^{*}$ \\
\hline Gamma & $32.7 \pm 4.9$ & $32.8 \pm 5.0$ & $31.4 \pm 4.2$ & $31.9 \pm 5.0$ & $31.2 \pm 3.5$ & $31.3 \pm 3.9$ & $32.2 \pm 3.8$ & $32.6 \pm 3.9$ \\
\hline \multicolumn{9}{|l|}{ Free } \\
\hline Delta & $53.6 \pm 2.7$ & $53.6 \pm 2.5$ & $51.8 \pm 2.4$ & $52.0 \pm 2.5$ & $51.4 \pm 2.4$ & $51.7 \pm 2.4$ & $51.7 \pm 2.8$ & $51.7 \pm 2.8$ \\
\hline Theta & $48.1 \pm 2.6$ & $48.3 \pm 2.4$ & $47.4 \pm 2.6$ & $47.4 \pm 2.7$ & $47.2 \pm 2.7$ & $47.4 \pm 2.7$ & $47.3 \pm 3.2$ & $47.3 \pm 3.3$ \\
\hline Alpha & $46.8 \pm 2.4$ & $46.9 \pm 2.4$ & $46.7 \pm 2.4$ & $46.9 \pm 2.5$ & $47.9 \pm 3.1$ & $48.2 \pm 3.0$ & $49.2 \pm 3.8$ & $49.2 \pm 3.9$ \\
\hline Beta & $41.2 \pm 2.5$ & $41.5 \pm 2.6$ & $40.8 \pm 2.3$ & $41.1 \pm 2.3$ & $40.8 \pm 2.2$ & $41.1 \pm 2.2$ & $41.2 \pm 2.4$ & $41.2 \pm 2.5$ \\
\hline Gamma & $34.0 \pm 6.3$ & $34.2 \pm 5.9$ & $33.4 \pm 6.2$ & $33.0 \pm 5.4$ & $32.0 \pm 4.2$ & $32.0 \pm 4.2$ & $32.4 \pm 3.7$ & $32.3 \pm 3.9$ \\
\hline
\end{tabular}

Each value represents the mean regional absolute power in $\mu \mathrm{V}^{2} \pm$ standard deviation.

AP: patients receiving any antipsychotic drug, Free: drug-free patients.

$p$ value represents the significance of differences in spectral power between drug-free patients and patients receiving any antipsychotic drug $(* p<$ $0.05 ; * * p<0.001)$. Mann - Whitney $U$ test. 
drug group did not significantly differ in sex, medication onset, disease onset, and disease classification (ICD-10). We found significant differences in GAF scores among patients in various antipsychotic drug groups with relatively low scores in the risperidone and olanzapine groups.

\section{Comparison of Spectral Power between the}

\section{Antipsychotic Drug Group and Drug-free Patients}

Compared with drug-free patients, patients taking any antipsychotic drug exhibited increases in theta and alpha power in all channels and increased beta power only in limited channels such as C3/C4 and P3/P4 (Table 2). In addition, we found no significant difference in gamma power between patients taking any antipsychotic drug and drug-free patients.

\section{Comparison of Spectral Power between Patients Taking} Each Type of Antipsychotic Drug and Drug-free Patients

Compared with drug-free patients, patients taking aripiprazole and blonanserin did not show a significant difference in any spectrum power (Table 3). In patients taking risperidone, we found significant increases in beta power

Table 3. Comparison of each spectral power between each antipsychotic drug group and drug-free patients

\begin{tabular}{|c|c|c|c|c|c|c|c|c|}
\hline Lead & F3 & $\mathrm{F} 4$ & $\mathrm{C} 3$ & $\mathrm{C} 4$ & P3 & P4 & O1 & $\mathrm{O} 2$ \\
\hline \multicolumn{9}{|l|}{ Free } \\
\hline Delta & $53.6 \pm 2.7$ & $53.6 \pm 2.5$ & $51.8 \pm 2.4$ & $52.0 \pm 2.5$ & $51.4 \pm 2.4$ & $51.7 \pm 2.4$ & $51.7 \pm 2.8$ & $51.7 \pm 2.8$ \\
\hline Theta & $48.1 \pm 2.6$ & $48.3 \pm 2.4$ & $47.4 \pm 2.6$ & $47.4 \pm 2.7$ & $47.2 \pm 2.7$ & $47.4 \pm 2.7$ & $47.3 \pm 3.2$ & $47.3 \pm 3.3$ \\
\hline Alpha & $46.8 \pm 2.4$ & $46.9 \pm 2.4$ & $46.7 \pm 2.4$ & $46.9 \pm 2.5$ & $47.9 \pm 3.1$ & $48.2 \pm 3.0$ & $49.2 \pm 3.8$ & $49.2 \pm 3.9$ \\
\hline Beta & $41.2 \pm 2.5$ & $41.5 \pm 2.6$ & $40.8 \pm 2.3$ & $41.1 \pm 2.3$ & $40.8 \pm 2.2$ & $41.1 \pm 2.2$ & $41.2 \pm 2.4$ & $41.2 \pm 2.5$ \\
\hline Gamma & $34.0 \pm 6.3$ & $34.2 \pm 5.9$ & $33.4 \pm 6.2$ & $33.0 \pm 5.4$ & $32.0 \pm 4.2$ & $32.0 \pm 4.2$ & $32.4 \pm 3.7$ & $32.3 \pm 3.9$ \\
\hline \multicolumn{9}{|l|}{ ARP } \\
\hline Delta & $52.9 \pm 2.2$ & $52.9 \pm 1.9$ & $52.2 \pm 2.0$ & $52.7 \pm 2.0$ & $52.8 \pm 2.0$ & $53.2 \pm 2.0$ & $53.8 \pm 3.0$ & $53.4 \pm 2.5$ \\
\hline Theta & $50.0 \pm 2.6$ & $50.2 \pm 2.6$ & $49.8 \pm 2.6$ & $50.2 \pm 2.9$ & $50.0 \pm 2.8$ & $50.2 \pm 3.1$ & $50.6 \pm 3.4$ & $50.3 \pm 3.7$ \\
\hline Alpha & $49.3 \pm 3.4$ & $49.4 \pm 3.2$ & $49.4 \pm 3.2$ & $49.7 \pm 3.2$ & $50.5 \pm 3.6$ & $50.4 \pm 3.8$ & $52.3 \pm 4.7$ & $52.0 \pm 5.1$ \\
\hline Beta & $41.1 \pm 1.7$ & $41.2 \pm 1.7$ & $40.9 \pm 1.6$ & $41.2 \pm 1.8$ & $41.2 \pm 2.0$ & $41.4 \pm 2.0$ & $42.1 \pm 3.0$ & $41.9 \pm 3.3$ \\
\hline Gamma & $29.9 \pm 3.8$ & $31.2 \pm 3.9$ & $29.1 \pm 3.2$ & $29.6 \pm 3.6$ & $29.0 \pm 2.8$ & $29.1 \pm 3.1$ & $29.5 \pm 2.9$ & $29.8 \pm 3.4$ \\
\hline \multicolumn{9}{|l|}{ RIS } \\
\hline Delta & $51.5 \pm 2.7$ & $51.4 \pm 2.2$ & $50.4 \pm 1.4$ & $50.4 \pm 1.0$ & $50.1 \pm 1.0$ & $50.4 \pm 1.2$ & $50.1 \pm 1.8$ & $50.5 \pm 2.3$ \\
\hline Theta & $49.4 \pm 1.5$ & $49.2 \pm 1.4$ & $49.2 \pm 1.4$ & $49.0 \pm 1.3$ & $49.0 \pm 1.6$ & $49.1 \pm 1.5$ & $48.7 \pm 2.2$ & $49.6 \pm 2.6$ \\
\hline Alpha & $49.6 \pm 2.4$ & $49.7 \pm 2.5$ & $50.3 \pm 2.5^{*}$ & $50.6 \pm 2.6^{*}$ & $51.6 \pm 2.5$ & $51.8 \pm 2.7$ & $52.1 \pm 3.0$ & $52.9 \pm 3.1$ \\
\hline Beta & $43.9 \pm 2.6$ & $44.0 \pm 2.7$ & $44.1 \pm 2.1^{*}$ & $44.7 \pm 2.4^{*}$ & $44.2 \pm 2.3^{*}$ & $44.3 \pm 2.2^{*}$ & $43.5 \pm 2.6$ & $43.9 \pm 2.6$ \\
\hline Gamma & $35.2 \pm 3.7$ & $35.4 \pm 5.3$ & $33.3 \pm 3.9$ & $35.7 \pm 6.1$ & $32.7 \pm 2.9$ & $33.6 \pm 3.8$ & $33.4 \pm 2.7$ & $33.7 \pm 3.0$ \\
\hline \multicolumn{9}{|l|}{ CLZ } \\
\hline Delta & $55.7 \pm 1.9$ & $55.1 \pm 2.5$ & $54.2 \pm 1.5$ & $53.9 \pm 1.6$ & $54.0 \pm 1.9$ & $54.1 \pm 2.2$ & $52.1 \pm 3.2$ & $52.0 \pm 2.9$ \\
\hline Theta & $53.9 \pm 2.6$ & $53.6 \pm 3.0$ & $52.7 \pm 2.8$ & $52.9 \pm 2.9 *$ & $51.7 \pm 2.6^{*}$ & $52.1 \pm 3.1^{*}$ & $49.9 \pm 3.2$ & $49.1 \pm 4.1$ \\
\hline Alpha & $48.2 \pm 1.9$ & $47.9 \pm 1.3$ & $47.8 \pm 1.4$ & $48.0 \pm 1.2$ & $48.1 \pm 1.2$ & $47.8 \pm 1.2$ & $47.3 \pm 1.8$ & $46.1 \pm 1.9$ \\
\hline Beta & $39.5 \pm 3.4$ & $39.6 \pm 2.7$ & $39.4 \pm 2.8$ & $39.8 \pm 2.5$ & $39.2 \pm 2.2$ & $39.4 \pm 2.0$ & $38.8 \pm 2.0$ & $38.9 \pm 2.0$ \\
\hline Gamma & $27.3 \pm 2.9$ & $27.5 \pm 2.3$ & $27.0 \pm 2.6$ & $26.9 \pm 2.1$ & $28.3 \pm 2.9$ & $27.8 \pm 2.2$ & $29.1 \pm 3.4$ & $30.8 \pm 4.0$ \\
\hline \multicolumn{9}{|l|}{ OLZ } \\
\hline Delta & $59.1 \pm 4.2$ & $58.8 \pm 3.8$ & $56.8 \pm 4.5$ & $57.0 \pm 4.3$ & $56.8 \pm 3.9^{*}$ & $57.0 \pm 3.5^{*}$ & $56.7 \pm 4.7$ & $57.0 \pm 5.0$ \\
\hline Theta & $53.0 \pm 3.4$ & $52.5 \pm 3.2$ & $51.6 \pm 2.5$ & $51.4 \pm 2.9$ & $51.0 \pm 3.2$ & $51.0 \pm 3.2$ & $50.6 \pm 3.8$ & $50.4 \pm 4.1$ \\
\hline Alpha & $50.3 \pm 4.9$ & $50.2 \pm 4.5$ & $50.0 \pm 3.8$ & $50.0 \pm 3.9$ & $50.6 \pm 5.5$ & $50.3 \pm 5.1$ & $51.2 \pm 6.1$ & $51.1 \pm 6.6$ \\
\hline Beta & $44.7 \pm 3.6$ & $44.8 \pm 3.5$ & $44.6 \pm 3.5$ & $44.3 \pm 3.2$ & $44.5 \pm 3.8$ & $44.2 \pm 3.7$ & $44.9 \pm 3.6$ & $45.1 \pm 3.9$ \\
\hline Gamma & $37.0 \pm 4.5$ & $36.1 \pm 4.8$ & $35.2 \pm 6.2$ & $34.0 \pm 6.4$ & $34.0 \pm 3.9$ & $33.8 \pm 4.2$ & $35.3 \pm 3.8$ & $36.0 \pm 3.8$ \\
\hline \multicolumn{9}{|l|}{ BNS } \\
\hline Delta & $55.4 \pm 3.9$ & $55.3 \pm 4.5$ & $53.6 \pm 5.0$ & $53.4 \pm 5.0$ & $53.7 \pm 3.8$ & $53.9 \pm 4.4$ & $54.3 \pm 5.9$ & $53.7 \pm 5.2$ \\
\hline Theta & $50.4 \pm 3.5$ & $50.6 \pm 3.4$ & $49.7 \pm 3.9$ & $49.6 \pm 3.6$ & $49.5 \pm 3.8$ & $49.6 \pm 4.1$ & $49.6 \pm 5.6$ & $49.3 \pm 5.3$ \\
\hline Alpha & $49.1 \pm 2.7$ & $49.3 \pm 3.0$ & $49.4 \pm 2.8$ & $49.2 \pm 2.7$ & $51.3 \pm 3.7$ & $50.8 \pm 3.6$ & $53.9 \pm 5.5$ & $53.0 \pm 5.1$ \\
\hline Beta & $42.0 \pm 2.7$ & $42.0 \pm 2.7$ & $42.2 \pm 2.7$ & $42.0 \pm 2.9$ & $43.5 \pm 2.9$ & $42.9 \pm 3.0$ & $44.7 \pm 4.4$ & $44.5 \pm 3.9$ \\
\hline Gamma & $33.6 \pm 1.9$ & $32.4 \pm 1.3$ & $32.1 \pm 1.2$ & $31.8 \pm 1.4$ & $32.4 \pm 2.0$ & $31.9 \pm 1.9$ & $34.4 \pm 2.7$ & $33.9 \pm 2.9$ \\
\hline
\end{tabular}

Each value represents the mean regional absolute power in $\mu \mathrm{V}^{2} \pm$ standard deviation.

Free: drug-free patients. ARP, aripiprazole; RIS, risperidone; CLZ, clozapine; OLZ, olanzapine; BNS, blonanserin. $p$ value represents the significance of differences in spectral power between drug-free patients and each antipsychotic drug group $\left({ }^{*} p<0.05\right)$. A Mann-Whitney $U$ test with a Dunn correction were used to adjust the $p$ values for multiple comparisons. 


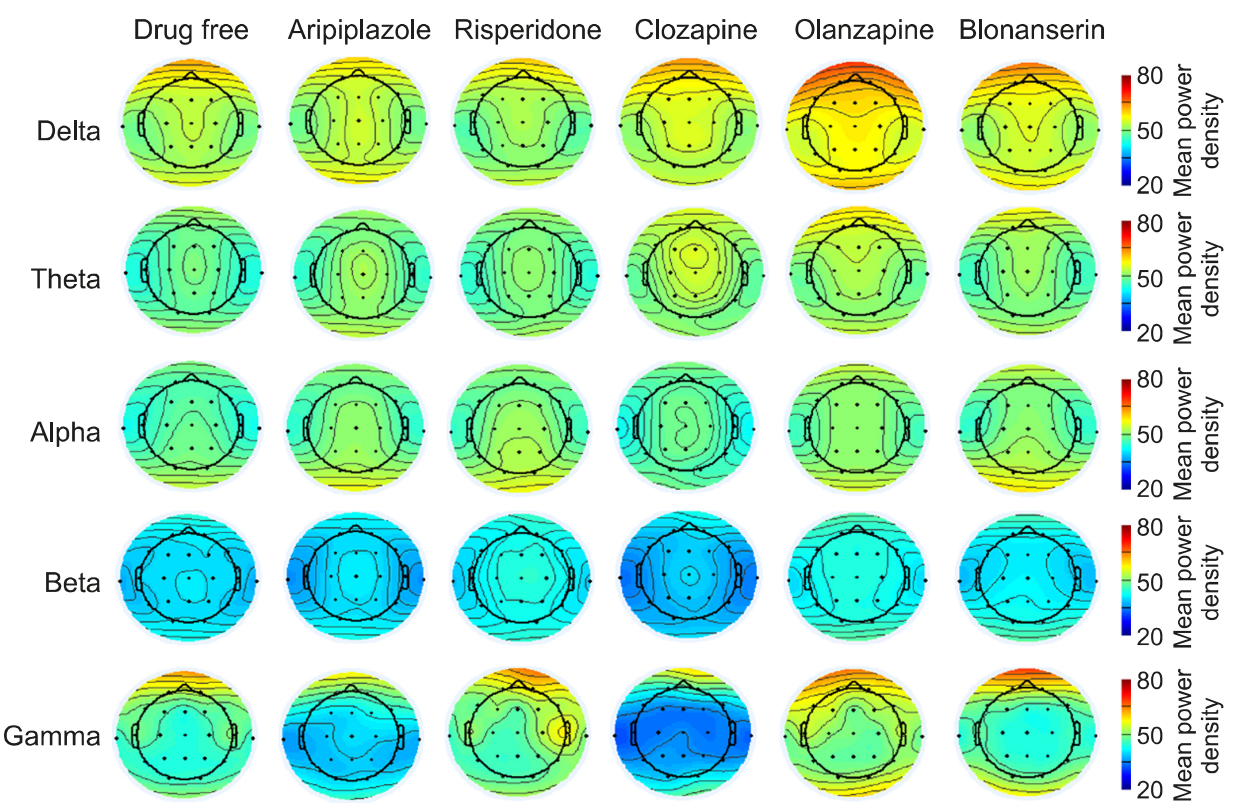

Fig. 1. Topographical maps of power spectral density of delta, theta, alpha, beta and gamma frequency bands in groups receiving antipsychotic drug monotherapy and without drug among schizophrenia spectrum disorders.

Table 4. Multiple regression analysis of spectral power and the equivalent dose of chlorpromazine

\begin{tabular}{cccccccc}
\hline & \multicolumn{3}{c}{ Theta } & & \multicolumn{2}{c}{ Alpha } \\
\cline { 2 - 3 } CP eq. & $\begin{array}{c}\text { Partial correlation } \\
\text { coefficient }\end{array}$ & Standardized $\beta$ & $p$ value & $\begin{array}{c}\text { Partial correlation } \\
\text { coefficient }\end{array}$ & Standardized $\beta$ & $p$ value \\
\hline F3 & 0.367 & 0.327 & $0.011^{*}$ & 0.325 & 0.263 & 0.087 \\
F4 & 0.329 & 0.272 & $0.019^{*}$ & 0.304 & 0.230 & 0.134 \\
C3 & 0.445 & 0.379 & $0.008^{*}$ & 0.372 & 0.265 & 0.066 \\
C4 & 0.342 & 0.290 & $0.009^{*}$ & 0.316 & 0.247 & 0.104 \\
\hline
\end{tabular}

$\mathrm{CP}$ eq., equivalent dose of chlorpromazine.

${ }^{*} p<0.05$.

in the $\mathrm{C} 3 / \mathrm{C} 4$ and $\mathrm{P} 3 / \mathrm{P} 4$ channels and in alpha power in the $\mathrm{C} 3 / \mathrm{C} 4$ channel. In patients taking clozapine, we found a significant increase in theta power mainly in the P3/P4 channel. In patients taking olanzapine, significant increases in delta power were found in the $\mathrm{C} 3 / \mathrm{C} 4$ and $\mathrm{P} 3 / \mathrm{P} 4$ channels along with a significant increase in the theta power of the F3 channel. We also have created topographical maps of power spectral density of delta, theta, alpha, beta and gamma frequency bands in groups receiving antipsychotic drug monotherapy and without drug (Fig. 1).

\section{Association between Any Band Power and the CP eq. for All Patients}

Pearson's correlation analysis showed that power in the alpha and theta bands had a weak positive correlation with the CP eq. in the C3 (alpha: rho 0.29, theta: rho 0.31), C4 (alpha: rho 0.27, theta: rho 0.30), and F3 (alpha: rho 0.29 , theta: rho 0.30 ) channels.

A multiple regression model including age, sex, and disease onset revealed that $\mathrm{CP}$ eq. was significantly associated with theta band power in the F3/F4 and C3/C4 channels (Table 4). However, the association between alpha band power and the CP eq. was not significant after adjusting for confounding factors.

\section{DISCUSSION}

In this retrospective cross-sectional study, we investigated the effect of antipsychotic drugs on quantitative 
EEG data in patients with schizophrenia by comparing patients using monotherapy and drug-free patients. Specifically, we aimed to evaluate the exact effect of antipsychotic drugs on quantitative EEG findings in patients undergoing strict monotherapy with approximately equal demographic conditions. As a result, we found that compared with drug-free patients, patients using any antipsychotic drug were associated with significant increases mainly in theta and alpha power. In particular, the CP eq. was independently associated with theta band power in the F3/F4 and C3/C4 channels after adjusting for sex, age, and disease onset. This finding suggests a dose-dependent effect of antipsychotic drugs on theta power. Compared with drug-free patients, each type of antipsychotic drug was associated with a different band power. Thus, each drug may have a different effect on quantitative EEG results based on its pharmacological characteristics.

Antipsychotic treatment is known to induce EEG modifications, mainly including a general slowing of the background activity, i.e., an increase in theta or delta activity [26]. Several quantitative studies have shown that lowpotency neuroleptics induce increases in theta power, while high-potency neuroleptics induce increases in alpha and beta power $[1,14,15]$. Tislerova reported that the use of atypical antipsychotics was associated with increases in delta and theta power in the fronto/temporo/occipital region [17]. Our finding that use of any antipsychotic drug was associated with a significant increase mainly in theta power is consistent with previous reports.

When each type of antipsychotic drug was considered, the increases in theta or delta power in the clozapine and olanzapine groups were more pronounced than those observed for other drugs in our study. This finding is also consistent with previous reports showing that clozapine and olanzapine were associated with increases in theta or delta power in the frontal area $[17,18]$. Additionally, clozapine and olanzapine have been shown to act on various receptor types. Both drugs show weak affinity for dopamine D2 receptors and an affinity for other systems, such as the cholinergic, histaminergic, serotonin $1 \mathrm{~A}\left(5-\mathrm{HT}_{1 \mathrm{~A}}\right)$, and $5-\mathrm{HT}_{2 \mathrm{c}}$ systems [27], improving positive, negative, and depressive symptoms among patients with schizophrenia [28]. In addition, the drug action of clozapine was previously found to be related to an increase in fast alpha power, which may also predict its therapeutic effectiveness [7]. In our study, no significant change was ob- served in alpha power, possibly because we did not separate patients into treatment response/non-response groups.

Moreover, a previous report further suggested that clozapine, amitriptyline, and zotepine, which are all chemically considered to be tricyclic derivatives, are associated with increases in delta and theta power [12]. Our results are consistent with this report in that olanzapine and clozapine showed similar quantitative EEG alterations. In general, drugs with similar therapeutic effects have similar effects on quantitative EEG profiles. In addition to this idea, our findings suggested that the chemical/physical characteristics of each compound may have important effects on the quantitative EEG results.

Regarding the effect of risperidone on quantitative EEG results, many previous studies have focused on healthy subjects, resulting in an increase in delta and theta or alpha power $[5,11]$. Our results were partially consistent with these results in that risperidone is associated with increases in alpha and beta power. However, other studies have suggested that risperidone does not affect any spectral power $[9,17]$. Thus, the effect of risperidone on quantitative EEG needs further to be investigated.

Only a few studies have examined the effect of aripiprazole on quantitative EEG data. In a clinical trial that recruited five patients with schizophrenia, EEG spectral analysis was performed and compared with the results of healthy controls, which revealed no significant difference in any band power [29]. In another study, pharmacodynamic analysis suggested that the concentration trends of aripiprazole were associated with delta activity only in patients with specific CYP2D6 genotypes [10]. Our results showed that aripiprazole is not associated with any spectral band power, possibly because our patients were heterogeneous and many did not have specific CYP2D6 genotypes, which may have affected the results.

To the best of our knowledge, no report has examined the effect of blonanserin on quantitative EEG results. Blonanserin is a relatively new antipsychotic that is widely used in eastern Asian countries [21]. Blonanserin is an unique atypical antipsychotic drug in that it has a high affinity for D2 rather than $5-\mathrm{HT}_{2 \mathrm{~A}}$ receptors [21]. In our study, similar to aripiprazole, blonanserin did not exhibit any relationship with any spectrum band power, possibly because its chemical structure and pharmacological profile are unique among atypical antipsychotic drugs.

Recently, gamma oscillation was proposed to play a 
fundamental role in basic aspects of information processing during sensory processing and higher cognitive functions [30]. Our study found no association between antipsychotic drugs and gamma band power, which is consistent with previous reports on patients with schizophrenia in a resting state condition with their eyes closed $[4,31,32]$. One of the few studies that performed resting state gamma band power analysis suggested that patients with schizophrenia show higher gamma band power in the left parietal and temporal area than healthy controls in the initial state. However, administration of atypical antipsychotics did not cause any significant change [4]. Another study using multiscale entropy analysis suggested that use of any psychotropic drug did not affect gamma power [31]. Other researchers using a time and domain frequency analysis method, called global field synchronization, suggested that the gamma global field synchronization is unaffected by antipsychotic treatment [32]. However, recent studies using auditory steady-state responses demonstrated that the use of atypical antipsychotics normalizes low gamma-evoked oscillations [20]. Hence, atypical antipsychotics have the potential to influence gamma activity impairment in patients with schizophrenia only under limited conditions, whereas under resting state conditions, atypical antipsychotics do not have any effect on gamma oscillations. This finding suggests that abnormalities in resting state gamma power may be another trait marker of schizophrenia, as noted previously [4].

Finally, we found that the CP eq. exhibited a weak positive correlation with theta power, and thus the use of atypical antipsychotic drugs may have a dose-dependent effect on theta power. This result is consistent with a previous study that found that the blood concentration of haloperidol was positively correlated with the theta power, especially in the group in which the medication was effective [13]. Although we did not obtain any data on the blood concentrations of antipsychotic drugs, we speculate that the blood concentration rather than the dose of antipsychotics is more closely associated with the EEG spectral power according to a previous paper. A similar finding was noted for the relationship between the blood concentration of clozapine and grand mal seizure [33].

This study has some important limitations. It is a cross-sectional study and therefore does not allow any causal relationships to be determined. Our sample size was small because of the strict inclusion and exclusion criteria, although similar sample sizes have been reported in previous studies $[4,7,17]$. Only drug-free patients were enrolled as a control group, and a healthy control group was not included. Therefore, it may be difficult to distinguish drug-induced modifications of EEG results from disease-induced results. The patient sample was heterogenous and included patients with conditions ranging from acute to chronic schizophrenia. Our data were extracted from single center, which may affect the generalizability of the study. Despite these limitations, our report is one of the few studies describing the effect of antipsychotic drug monotherapy on quantitative EEG results, including the gamma power band, in patients with schizophrenia.

In summary, our result showing that antipsychotic use is associated with an increase in theta power is consistent with previous reports. We also found a dose-dependent relationship in the increase in theta power, which may be a common characteristic of atypical antipsychotic drugs. We did not find any change in gamma power, which may confirm the hypothesis that resting state gamma power is a trait marker of schizophrenia. Comparison of different antipsychotic drugs suggested that these drugs had varying effects on quantitative EEG results based on their pharmacological characteristics and chemical structures. Of note, this study focused on the effects of atypical antipsychotic drugs on quantitative EEG results among patients with schizophrenia, which revealed not only their common features but also their singular electrophysiological properties. Similar to the results of the blonanserin group in our study, a quantitative EEG approach for evaluation of new drugs may provide insight into their pharmacological and chemical properties.

\section{Acknowledgments}

We thank everyone who participated in this study. We are grateful to the psychiatrists who discussed the findings with the authors. We thank Lisa Kreiner, PhD, from Edanz Group (https://en-author-services.edanzgroup.com/) for editing a draft of this manuscript.

\section{- Conflicts of Interest}

The authors report no financial or other relationship that is relevant to the subject of this article. IK has received honoraria from Astellas, Daiichi Sankyo, Dainippon 
Sumitomo Pharma, Eisai, Eli Lilly, Janssen Pharmaceutical, Kyowa Hakko Kirin, Lundbeck, Meiji Seika Pharma, MSD, Mylan, Novartis Pharma, Ono Pharmaceutical, Otsuka Pharmaceutical, Pfizer, Shionogi, Shire, Taisho Toyama Pharmaceutical, Takeda Pharmaceutical, Tanabe Mitsubishi Pharma, Tsumura, and Yoshitomiyakuhin, and has received research/grant support from Astellas, Daiichi Sankyo, Dainippon Sumitomo Pharma, Eisai, Eli Lilly, Kyowa Hakko Kirin, Mochida Pharmaceutical, MSD, Novartis Pharma, Otsuka Pharmaceutical, Pfizer, Shionogi, and Takeda Pharmaceutical. $\mathrm{NH}$ has received personal fees from Janssen Pharmaceutical, Yoshitomiyakuhin, Otsuka Pharmaceutical, Dainippon Sumitomo Pharma, Novartis Pharma, and Meiji Seika Pharma. TO and AT have no potential conflict of interest.

\section{Author Contributions}

Conceptualization: Takashi Ozaki, Naoki Hashimoto. Data acquisition: Takashi Ozaki, Atsuhito Toyomaki. Formal analysis: Takashi Ozaki, Atsuhito Toyomaki. Supervision: Ichiro Kusumi. Writing-original draft: Takashi Ozaki. Writing-review \& editing: Takashi Ozaki, Naoki Hashimoto.

\section{口 ORCID}

Takashi Ozaki

https://orcid.org/0000-0001-9930-9179

Atsuhito Toyomaki https://orcid.org/0000-0001-9333-8833

Naoki Hashimoto https://orcid.org/0000-0001-8311-7148

Ichiro Kusumi https://orcid.org/0000-0002-8747-5070

\section{REFERENCES}

1. Knott V, Labelle A, Jones B, Mahoney C. Quantitative EEG in schizophrenia and in response to acute and chronic clozapine treatment. Schizophr Res 2001;50:41-53.

2. John ER, Prichep LS, Alper KR, Mas FG, Cancro R, Easton P, et al. Quantitative electrophysiological characteristics and subtyping of schizophrenia. Biol Psychiatry 1994;36:801-826.

3. Newson JJ, Thiagarajan TC. EEG frequency bands in psychiatric disorders: a review of resting state studies. Front Hum Neurosci 2019;12:521.

4. Mitra S, Nizamie SH, Goyal N, Tikka SK. Evaluation of resting state gamma power as a response marker in schizophrenia. Psychiatry Clin Neurosci 2015;69:630-639.

5. Lee DY, Lee KU, Kwon JS, Jang IJ, Cho MJ, Shin SG, et al. Pharmacokinetic-pharmacodynamic modeling of risperidone effects on electroencephalography in healthy volunteers. Psychopharmacology (Berl) 1999;144:272-278.

6. Boutros NN, Arfken C, Galderisi S, Warrick J, Pratt G, lacono
W. The status of spectral EEG abnormality as a diagnostic test for schizophrenia. Schizophr Res 2008;99:225-237.

7. Mucci A, Volpe U, Merlotti E, Bucci P, Galderisi S. PharmacoEEG in psychiatry. Clin EEG Neurosci 2006;37:81-98.

8. Bewernitz M, Derendorf H. Electroencephalogram-based pharmacodynamic measures: a review. Int J Clin Pharmacol Ther 2012;50:162-184.

9. Allain H, Tessier C, Bentué-Ferrer D, Tarral A, Le Breton S, Gandon $\mathrm{M}$, et al. Effects of risperidone on psychometric and cognitive functions in healthy elderly volunteers. Psychopharmacology (Berl) 2003;165:419-429.

10. Kim E, Yu KS, Cho JY, Shin YW, Yoo SY, Kim YY, et al. Effects of DRD2 and CYP2D6 genotypes on delta EEG power response to aripiprazole in healthy male volunteers: a preliminary study. Hum Psychopharmacol 2006;21:519-528.

11. Romero S, Mañanas MA, Barbanoj MJ. Influence of ocular filtering in EEG data on the assessment of drug-induced effects on the brain. Hum Brain Mapp 2009;30:1470-1480.

12. Saletu B, Grünberger J, Linzmayer L, Anderer P. Comparative placebo-controlled pharmacodynamic studies with zotepine and clozapine utilizing pharmaco-EEG and psychometry. Pharmacopsychiatry 1987;20(1 Spec No):12-27.

13. Czobor $P$, Volavka J. Level of haloperidol in plasma is related to electroencephalographic findings in patients who improve. Psychiatry Res 1992;42:129-144.

14. Galderisi S, Mucci A, Mignone ML, Maj M, Kemali D. CEEG mapping in drug-free schizophrenics. Differences from healthy subjects and changes induced by haloperidol treatment. Schizophr Res 1991;6:15-23.

15. Kemali D, Galderisi S, Maj M, Mucci A, Di Gregorio M, Bucci P. Computerized EEG topography findings in schizophrenic patients before and after haloperidol treatment. Int J Psychophysiol 1992;13:283-290.

16. Small JG, Milstein V, Small IF, Miller MJ, Kellams JJ, Corsaro CJ. Computerized EEG profiles of haloperidol, chlorpromazine, clozapine and placebo in treatment resistant schizophrenia. Clin Electroencephalogr 1987;18:124-135.

17. Tislerova B, Brunovsky M, Horacek J, Novak T, Kopecek M, Mohr $\mathrm{P}$, et al. LORETA functional imaging in antipsychoticnaive and olanzapine-, clozapine- and risperidone-treated patients with schizophrenia. Neuropsychobiology 2008;58: 1-10.

18. Hyun J, Baik MJ, Kang UG. Effects of psychotropic drugs on quantitative EEG among patients with schizophrenia-spectrum disorders. Clin Psychopharmacol Neurosci 2011;9:78-85.

19. Uhlhaas PJ, Pipa G, Neuenschwander S, Wibral M, Singer W. A new look at gamma? High- $(>60 \mathrm{~Hz}) \gamma$-band activity in cortical networks: function, mechanisms and impairment. Prog Biophys Mol Biol 2011;105:14-28.

20. Alegre M, Molero P, Valencia M, Mayner G, Ortuño F, Artieda J. Atypical antipsychotics normalize low-gamma evoked oscillations in patients with schizophrenia. Psychiatry Res 2017;247:214-221. 
21. Tenjin T, Miyamoto S, Ninomiya Y, Kitajima R, Ogino S, Miyake $\mathrm{N}$, et al. Profile of blonanserin for the treatment of schizophrenia. Neuropsychiatr Dis Treat 2013;9:587-594.

22. World Health Organization. The ICD-10 classification of mental and behavioural disorders: diagnostic criteria for research. Geneva:World Health Organization; 1993.

23. Kay SR, Fiszbein A, Opler LA. The positive and negative syndrome scale (PANSS) for schizophrenia. Schizophr Bull 1987; 13:261-276.

24. Sinha SR, Sullivan L, Sabau D, San-Juan D, Dombrowski KE, Halford JJ, et al. American Clinical Neurophysiology Society guideline 1: minimum technical requirements for performing clinical electroencephalography. J Clin Neurophysiol 2016; 33:303-307.

25. $R$ Development Core Team. A language and environment for statistical computing [Internet]. Vienna: $R$ Foundation for Statistical Computing; 2015 [cited at 2020 Jun 25]. Available from: https://www.gbif.org/tool/81287/r-a-language-and-environment-for-statistical-computing.

26. Amann BL, Pogarell O, Mergl R, Juckel G, Grunze H, Mulert C, et al. EEG abnormalities associated with antipsychotics: a comparison of quetiapine, olanzapine, haloperidol and healthy subjects. Hum Psychopharmacol 2003;18:641-646.

27. Horacek J, Bubenikova-Valesova V, Kopecek M, Palenicek T, Dockery C, Mohr P, et al. Mechanism of action of atypical an- tipsychotic drugs and the neurobiology of schizophrenia. CNS Drugs 2006;20:389-409.

28. Huhn M, Nikolakopoulou A, Schneider-Thoma J, Krause M, Samara $M$, Peter $N$, et al. Comparative efficacy and tolerability of 32 oral antipsychotics for the acute treatment of adults with multi-episode schizophrenia: a systematic review and network meta-analysis. Lancet 2019;394:939-951.

29. Cañive JM, Lewine JD, Edgar JC, Davis JT, Miller GA, Torres F, et al. Spontaneous brain magnetic activity in schizophrenia patients treated with aripiprazole. Psychopharmacol Bull 1998;34:101-105.

30. Buzsáki G, Wang XJ. Mechanisms of gamma oscillations. Annu Rev Neurosci 2012;35:203-225.

31. Takahashi T, Cho RY, Mizuno T, Kikuchi M, Murata T, Takahashi $\mathrm{K}$, et al. Antipsychotics reverse abnormal EEG complexity in drug-naive schizophrenia: a multiscale entropy analysis. Neuroimage 2010;51:173-182.

32. Kikuchi M, Koenig T, Wada Y, Higashima M, Koshino Y, Strik $\mathrm{W}$, et al. Native EEG and treatment effects in neuroleptic-naïve schizophrenic patients: time and frequency domain approaches. Schizophr Res 2007;97:163-172.

33. Varma S, Bishara D, Besag FM, Taylor D. Clozapine-related EEG changes and seizures: dose and plasma-level relationships. Ther Adv Psychopharmacol 2011;1:47-66. 